Running Head: SENSITIVITY TO ENVIRONMENT

Preprint

\title{
People Differ in their Sensitivity to the Environment: An Integrated Theory, Measurement and Empirical Evidence
}

Michael Pluess ${ }^{1 * \#}$, Francesca Lionetti ${ }^{1,2^{*}}$, Elaine N. Aron ${ }^{3}$, Arthur Aron $^{3}$

${ }^{1}$ Department of Biological and Experimental Psychology, Queen Mary University of London, London, UK

${ }^{2}$ Department of Neurosciences, Imaging and Clinical Sciences, d'Annunzio University of Chieti-Pescara

${ }^{3}$ Department of Psychology, Stony Brook University, Stony Brook, USA

* Shared first authors

${ }^{\#}$ Correspondence to:

Michael Pluess

Department of Biological and Experimental Psychology

Queen Mary University of London

Mile End Road

London E1 4NS

United Kingdom

Email: m.pluess@qmul.ac.uk

Phone: +44 (0)207-882-8004 


\begin{abstract}
People differ in their response to similar experiences with some being generally more and some less sensitive. Several theories have been developed over the years to describe such differences in sensitivity and a growing number of studies in humans and other species provide empirical evidence that some individuals are indeed more affected than others by environmental influences as a function of various sensitivity markers. These include temperament traits in children as well as a number of biological factors such as physiological reactivity and genes. In this paper, we present an integrated theoretical perspective on Environmental Sensitivity and provide new empirical evidence that sensitivity, measured with a short questionnaire for adults, predicts differences in the response to negative as well as positive experiences. Results across four individual studies (total $N=1,140$ ) suggest that individual differences in sensitivity to the environment can be reliably and easily assessed with a short self-report measure (HSP-12), confirmed and validated by two empirical studies according to which the scale predicts heightened reactivity to both negative and positive experiences. Furthermore, our findings show that sensitivity reflects a continuous spectrum from low to high along which people fall into three distinct sensitivity groups (low, medium, and high), and that sensitivity is associated with a specific personality profile characterised by both high neuroticism and high openness to experiences. We conclude that empirical findings confirm the theoretical proposition that people differ in their sensitivity to environmental influences and propose a number of future directions to advance research on environmental sensitivity.
\end{abstract}

\title{
Keywords
}

Environmental Sensitivity; Sensory Processing Sensitivity; Highly Sensitive Person; Differential Susceptibility; Temperament; 


\section{People Differ in their Sensitivity to the Environment: An Integrated Theory,}

\section{Measurement and Empirical Evidence}

It is widely observable that humans differ considerably in their response to common experiences and environmental exposures with some being more affected than others. Such individual differences in sensitivity to the environment are supported by numerous empirical studies (for review, see Belsky \& Pluess, 2016; Obradovic \& Boyce, 2009; Pluess \& Belsky, 2010; Slagt et al., 2016) and have been conceptualised in several theoretical frameworks over the years (Aron \& Aron, 1997; Aron et al., 2012; Belsky, 1997; Belsky \& Pluess, 2009; Boyce \& Ellis, 2005; Ellis et al., 2011; Monroe \& Simons, 1991). More recently, the different theoretical perspectives have been integrated into the broader framework of Environmental Sensitivity according to which people vary in their general response to environmental influences due to differences in how they perceive and process information about the environment (Pluess, 2015). In this multi-study paper we describe the integrated framework of environmental sensitivity, introduce a brief self-report measure for sensitivity that is based on the original Highly Sensitive Person scale (Aron \& Aron, 1997), and investigate its psychometric properties as well as its relationship with the Big Five personality traits (including facets). We then explore the existence of different sensitivity groups and providing new evidence in two experimental studies that sensitivity measured with the new scale predicts differences in the response to negative as well as positive experiences.

\section{Sensitivity Theories}

The notion that people differ in their responsivity or reactivity to external stimuli as a function of behavioural traits is well established and recognised by several important psychological concepts and theories. This includes Kagan's influential work on inhibited temperament in children (Kagan, 1997; Kagan et al., 1987) as well as Gray's conceptualisation of personality as consisting of a behavioural inhibition and a behavioural 
activation system (Gray, 1981, 1982). Over the last two decades, several theories emerged that focus on differences in sensitivity to environmental influences more specifically. These include Sensory Processing Sensitivity (Aron \& Aron, 1997; Aron et al., 2012), Differential Susceptibility (Belsky, 1997; Belsky \& Pluess, 2009), and Biological Sensitivity to Context (Boyce, 1996; Boyce \& Ellis, 2005). The framework of Sensory Processing Sensitivity (Aron \& Aron, 1997; Aron et al., 2012) conceptualises sensitivity as a biologically-based and relatively stable temperament trait characterised by heightened sensory sensitivity, depth of processing, emotional/physiological reactivity, and behaviour inhibition. While most of the work on Sensory Processing Sensitivity has been conducted in adults, both Differential Susceptibility (Belsky, 1997; Belsky \& Pluess, 2009) and Biological Sensitivity to Context (Boyce, 1996; Boyce \& Ellis, 2005) are developmental concepts and informed by evolutionary theory. Differential Susceptibility (Belsky, 1997; Belsky \& Pluess, 2009) suggests that natural selection led to the development of two basic types of children: those that are genetically more plastic in their development, and others that are more fixed. The more plastic ones are more susceptible to environmental quality whilst growing up, and therefore more affected by both negative and positive environmental influences (Belsky et al., 2007). According to Biological Sensitivity to Context (Boyce, 1996; Boyce \& Ellis, 2005), sensitivity is primarily driven by physiological reactivity and shaped by the quality of the environment during childhood, with children growing up in particularly adverse or especially supportive contexts developing heightened sensitivity. Although these three theories differ in various ways, what they have in common is that they all propose that people differ in their sensitivity to environmental influences, and that more sensitive individuals are not only more reactive to adverse experiences, as the traditional Diathesis-Stress model suggests (Monroe \& Simons, 1991), but also particularly receptive to positive supportive ones - a claim that is supported by empirical evidence (see below). Importantly, the 
observation that people differ in their response to positive experiences has been conceptualised more specifically in the framework of Vantage Sensitivity (Pluess \& Belsky, 2013).

\section{Environmental Sensitivity: An Integrated Framework}

The various concepts and theories that describe individual differences in sensitivity to the environment have been integrated into the overarching umbrella framework of Environmental Sensitivity (Pluess, 2015; Pluess et al., 2018). According to the broad perspective of environmental sensitivity, people differ substantially in their sensitivity to negative and/or positive environmental influences with some generally more and others generally less sensitive. Sensitivity reflects the degree to which people are able to register detailed characteristics and changes in the physical or social environment, and cognitively process such sensory input in order to respond or react in ways that are adaptive given the specific features of the environment. Differences in sensitivity to environmental influences are understood to be the function of neurobiological mechanisms in the central nervous system (Ellis et al., 2011; Pluess, 2015) that influence an individual's capacity to perceive and process information about the environment, and are often reflected in heightened behavioural (e.g., inhibited temperament) and physiological reactivity (e.g., cortisol reactivity). In line with this hypothesis, several brain regions have been associated with sensitivity (Acevedo et al., 2014), including amygdala structure (Pluess et al., in press; Yap et al., 2008) and function (Gard et al., 2018) as well as hippocampus (Schriber et al., 2017; Whittle et al., 2011) and total brain volume (Nolvi et al., 2020). The framework of environmental sensitivity further suggests that differences in sensitivity have a genetic basis but are also shaped by experiences across development, including the prenatal period (Hartman et al., 2018; Pluess \& Belsky, 2011). According to a recent twin study, sensitivity assessed in a large sample of 17-year old adolescents with the Highly Sensitive Child scale 
(Pluess et al., 2018) has a substantial heritability of $47 \%$ with the remaining variance explained by environmental factors (Assary et al., 2020). Hence, genetic and environmental factors appear to contribute equally to the development of sensitivity. Whilst early molecular genetics studies on sensitivity focused primarily on the role of single candidate genes (Belsky et al., 2009), more recent genome-wide association studies (GWAS) suggest that sensitivity is the function of many thousands of gene variants across the genome, each making a small contribution rather than a few single genes with large effects (Keers et al., 2016), a discovery that is in line with other common human traits, such as the Big Five personality traits (Lo et al., 2016; Nagel et al., 2018; Power \& Pluess, 2015; Sanchez-Roige et al., 2018). Importantly, the integrated perspective of environmental sensitivity proposes that the degree and type of sensitivity is also shaped by the interaction between genetic and environmental factors across the life course. In more detail, whilst the quality of the environment tends to have a limited impact on the development of sensitivity in those with a low genetic predisposition for sensitivity (i.e., low sensitivity type regardless of environmental exposure), in those with high genetic sensitivity a more adverse background will result in particular sensitivity towards threat (i.e., vigilant sensitivity type) (Kumsta et al., 2010; Pluess et al., 2010), a history of supportive care contribute to the development of particular sensitivity to positive experiences (i.e., vantage sensitivity type) (Drury et al., 2012), and equal measures of adversity and support result in a generally high sensitivity type (Drury et al., 2012; Pluess, 2015). For example, in a large longitudinal study from birth to 50 years of age, those with high genetic sensitivity, assessed with a candidate gene polygenic score of sensitivity, developed higher vulnerability to low environmental quality as adults when growing up in low quality settings, but also more resilience to adversity in adulthood when having grown up in high quality environment (Keers \& Pluess, 2017). According to evolutionary considerations, both low and high sensitivity have specific advantages and disadvantages and therefore reflect different but 
equally valuable developmental strategies. Whilst less sensitive individuals tend to be more robust and resilient when faced with adversity, they are somewhat less likely to register and benefit from opportunities. More sensitive individuals, on the other hand, may recognise and absorb positive influences more easily and strongly, but are also more vulnerable to the negative effects of adverse experiences. However, although both low and high environmental sensitivity can be beneficial from an evolutionary perspective, according to several empirical studies and computer simulation, high sensitivity is most advantageous if characterizing a minority of the population (about 20-30\%; Wolf et al., 2008).

\section{Empirical Evidence for Individual Differences in Environmental Sensitivity}

A growing number of studies across multiple fields in psychology provide empirical evidence for individual differences in environmental sensitivity (e.g., Belsky \& Pluess, 2009, 2011, 2013, 2016; Greven et al., 2019; Obradovic \& Boyce, 2009; Pluess \& Belsky, 2010; Slagt et al., 2016). For example, the study of temperament-parenting interactions has a long history in developmental psychology and findings generally suggest that the effects of parenting behaviours on child development vary as a function of infant temperament. According to a recent meta-analysis based on 84 temperament-parenting interaction studies, many of the detected interaction effects were consistent with environmental sensitivity in that children with certain temperament traits that capture aspects of heightened sensitivity (i.e., difficult temperament, negative emotionality) are more negatively affected by harsh parenting practices but also respond disproportionately positively to particularly supportive care (Slagt et al., 2016). Similarly, in the field of clinical psychology, theory suggests that people characterised by an inherent vulnerability for psychopathology (e.g., genetic predisposition for mental health problems) are more likely to develop psychological problems when facing adversity (Monroe \& Simons, 1991). Interestingly, several putative vulnerability genes have been shown to be associated not only with an increased risk for the development of problems 
in response to adversity, but also with an attenuated risk when experiencing a supportive environment (Bakermans-Kranenburg \& van IJzendoorn, 2011; Belsky et al., 2009; van IJzendoorn et al., 2012). Importantly, genetic evidence is not restricted to candidate gene studies which have been criticised for a number of important methodological issues (Duncan \& Keller, 2011). Individual differences in genetic sensitivity to both negative and positive experiences have also been observed in studies featuring genome-wide approaches such as polygenic scores based on thousands of genetic variants (Keers et al., 2016; Pluess et al., submitted). However, although the growing evidence base for environmental sensitivity is providing a strong foundation, additional work is required to address a number of important issues. For example, much of the evidence is based on correlational designs rather than experimental studies that manipulate the environmental exposure, predominately focused on children and adolescents, and sensitivity is often indicated by relatively distal markers of sensitivity (i.e., difficult temperament, physiological reactivity, genetic variants) rather than sensitivity behaviours measured more specifically and directly.

\section{Phenotype of Environmental Sensitivity}

Self-reported sensitivity in adults can be measured with the Highly Sensitive Person (HSP) scale which has been developed almost 25 years ago based on a series of interviews with people that self-identified as being highly sensitive, and then systematically validated over several studies (Aron \& Aron, 1997). The scale consists of 27 items and according to a recent analysis fits a bifactor structure with items loading onto one general sensitivity factor as well as three individual factors that capture different aspects of sensitivity (Lionetti et al., 2018) - rather than all items loading onto a single factor as initially proposed (Aron \& Aron, 1997). More recently, a range of new measures have been developed to assess sensitivity in children, including the 12-item Highly Sensitive Child (HSC) scale (Pluess et al., 2018) and the Highly Sensitive Child-Rating System (HSC-RS) (Lionetti, Aron, et al., 2019), an 
objective observer-rated measure of sensitivity in young children. According to longitudinal and experimental studies, children that score high on these measures have been found to be more affected by their experiences such as school-based psychological programmes (Nocentini et al., 2018; Pluess \& Boniwell, 2015) and parenting quality (Lionetti, Aron, et al., 2019; Slagt et al., 2018).

\section{Limitations of Existing Knowledge}

However, whilst a substantial number of studies provide evidence that children differ in their sensitivity to both negative and positive experiences, more work is needed to investigate sensitivity in adults, including the improvement and validation of sensitivity measures in experimental studies. Hence, the current paper includes the development of an adult HSP scale that is short and easy to complete, yet psychometrically strong. According to a recent meta-analysis on the relationship between sensitivity measures and the Big Five personality traits, sensitivity assessed with the HSP and HSC scales is significantly and positively associated with neuroticism and openness to experiences but not extraversion, agreeableness, or conscientiousness (Lionetti, Pastore, et al., 2019). However, a more detailed investigation of the association with common personality traits, including at the facet level, is important in order to get a clearer picture of the personality profile underlying sensitivity to environmental influences in adults (for a recent paper on Big Five facets and sensitivity, see Bröhl et al., 2020). It also remains to be tested whether sensitivity measured with the HSP scale can be clearly differentiated from associated personality traits and their facets. Furthermore, the original theoretical proposition that about $20 \%$ of the general population are characterised by high sensitivity in contrast to the remaining $80 \%$ that are not (Aron et al., 2012; Boyce \& Ellis, 2005), has recently been challenged by several studies suggesting that sensitivity is a normal distributed trait along which people fall into three, rather than two, distinct sensitivity groups from low (30\%), to medium (40\%), to high (30\%) (Lionetti et al., 
2018; Pluess et al., 2018). It is important to investigate whether the brief HSP scale presented in this paper supports the same three-group solution in an adult population. Finally, the fundamental propositions of the environmental sensitivity framework can only be confirmed if sensitivity assessed with the sensitivity scale is actually predicting the response to both negative and positive experiences which requires experimental rather than correlational studies.

\section{Aims and Hypotheses of the Current Paper}

The current paper aims to address all these points across four studies with a total combined sample size of $N=1,140$. The first study (Study 1 ) introduces a short version of the original Highly Sensitive Person (HSP) scale, followed by the investigation of associations with the Big Five personality dimensions and facets. Study 2 focuses on testing for the existence of low, medium, and high sensitivity groups with the help of Latent Class Analysis. Studies 3 and 4 feature experimental designs and focus on testing whether sensitivity measured with the brief HSP scale predicts people's responses to environmental influences. Individual differences in sensitivity to negative experiences are tested in a single-group experimental study in a sample of teacher trainees that are teaching for the first time after minimal training (Study 3), and differences in response to positive experiences in a lab-based mood induction experiment using a video with uplifting content (Study 4).

Based on theory and existing research, we hypothesise that (a) sensitivity measured with the brief version of the HSP scale is associated with the dimensions and facets of neuroticism and openness to experiences but distinguishable from these established traits, (b) three sensitivity groups (low, medium, high) emerge in Latent Class Analysis, (c) sensitive teachers are more negatively affected by their first-time teaching experience, and (d) sensitive individuals' happiness is more easily influenced by positive mood induction.

\section{Study 1}


The first objective of Study 1 was to create a short and psychometrically robust version of the original 27-item Highly Sensitive Person (HSP-27) scale (Aron \& Aron, 1997) that is widely applicable. Although the HSP-27 was initially designed to reflect one single factor, follow-up studies revealed that the 27 -item scale fitted a bifactor structure best (Lionetti et al., 2018), characterised by one general and three individual factors (Ease of Excitation [EOE], Low Sensory Threshold [LST], and Aesthetic Sensitvity [AES] according to Smolewska et al., 2006). We aimed to create and test a brief version of the scale that retains the recently identified bifactor structure by selecting those items that load strongest on the three individual factors and are most likely applicable across different cultures (the original scale was developed in an exclusively U.S. sample).

The second objective of the study was to investigate how the newly developed brief HSP scale relates to the Big Five personality traits at the factor as well as at the facet level. According to a recent meta-analysis of previous studies featuring the HSP-27 and the related 12-item Highly Sensitive Child (HSC) scale (Pluess et al., 2018), sensitivity correlated significantly with neuroticism $(r=.40)$ and openness $(r=.14)$ but not with extraversion, agreeableness or conscientiousness (Lionetti, Pastore, et al., 2019). However, the majority of existing studies primarily investigated associations between the sensitivity measures and the five personality dimensions not taking into account how the total score and the three subscales of the sensitivity measures relate to the various facets of the Big Five personality traits (for a first exploration of the facets in relation to sensitivity, see Bröhl et al., 2020). Hence, we collected data on personality traits at the facet level in order to investigate how environmental sensitivity relates to established personality traits and their facets as well as to test divergent validity of those sensitivity components that correlate highly with personality traits. Based on previous research, we expected that EOE and LST would correlate 
moderately with neuroticism and all its facets, and AES moderately with openness to experiences and all its facets.

\section{Methods}

Participants. We recruited a sample of 722 adults (mean age $=33.5$ years, $S D=8.1$, 66.2\% female) through Prolific, an online survey service which offers interested volunteers participation in research studies in exchange for small monetary compensation. Inclusion criteria required that participants were: 1) living in the United Kingdom, and 2) between 2050 years old. Ethnic distribution of the sample was as follows: $89.8 \%$ Caucasian, $5.1 \%$ AsianIndian, $1.8 \%$ African/Caribbean, .3\% Arab, 2.9\% mixed (one participant chose not to answer). The study was approved by the institutional ethics committee (Queen Mary University of London).

Procedure and Development of the brief HSP Scale. In order to identify the most suitable items for inclusion in the brief HSP scale, we considered Smolewska et al.'s (2006) factor analysis solution of the 27-item HSP scale which featured a sample of 851 Canadian psychology students (mean age $=19.7$ years, $S D=2.9$ years, $68.8 \%$ female). We aimed to select items that best represented each of the three detected factors (EOE, AES and LST) according to their factor loadings (only items with factor loadings of $>.50$ or higher were considered) whilst also being relevant and meaningful in non-North American and potentially non-Western cultures. Furthermore, we tried to retain a similar proportional representation of the subscales as in the 27-item version (i.e., approx. $48 \%$ of items reflecting EOE, $28 \%$ AES, and 24\% LST). Taking all these aspects into considerations, we ended up with a brief HSP scale that features 12 of the original items (HSP-12), including 5 items for EOE, 4 items for AES and 3 items for LST (see Table 1 for a list of the selected items and the subscale they belong to). 
We then asked participants, after they provided informed consent, to complete an online survey, which included basic demographic information, the newly developed HSP-12 scale, and a measure for the Big Five personality traits.

$$
\begin{aligned}
& \text {--- Insert Table } 1 \text { here --- } \\
& \text {--- Insert Figure } 1 \text { here--- }
\end{aligned}
$$

Measures. The original Highly Sensitive Person (HSP) scale consists of 27 items (Aron \& Aron, 1997) which state questions that participants rate on a 7-point Likert-type scale from $1=$ "Not at all" to $7=$ "Extremely" to the extent that the item applies to them (e.g., "Are you deeply moved by the arts or music?", "Do you seem to be aware of subtleties in your environment?”, “Are you bothered by intense stimuli, like loud noises or chaotic scenes? "). In this study, sensitivity was measured with the newly developed HSP-12 scale which is based on 12 items (see Table 1) from the original 27-item scale, rated on the same 7point Likert scale. Items are averaged to obtain a total mean score.

The Big Five personality traits (extraversion, neuroticism, agreeableness, conscientiousness and openness to experiences) and associated facets were assessed with 120 items from the freely and publicly available International Personality Item Pool (IPIP) (Goldberg, 1999). Items contain phrases describing specific behavior at trait and facet level (e.g., “Worry about things” for neuroticism, "Love large parties” for extraversion, "Trust others" for agreeableness, "Like to tidy up" for conscientiousness, and "Love to read challenging material" for openness to experiences) which participants rated on a 5-point Likert scale ranging from 1 = "Very Inaccurate" to 5 = "Very Accurate" based on how well items described them (Johnson, 2014). Each of the five personality dimensions was assessed with a total of 24 items which are divided further into six facets for each dimension (i.e., 4 
items per facet). In the current sample the five personality dimensions had good internal consistency with Cronbach's $\alpha=.91$ for neuroticism, $\alpha=.90$ for extraversion, $\alpha=.81$ for openness to experiences, $\alpha=.89$ for conscientiousness, and $\alpha=.88$ for agreeableness.

Data Analysis. First, we considered the HSP-12 total mean score and its distribution. Second, we investigated the degree of correlation between the newly developed HSP-12 with the HSP-27 item version using data from a previous study where participants $(N=906)$ completed the longer 27-item version (Lionetti et al., 2018). Then, we applied a Confirmatory Factor Analysis (CFA) to investigate whether the selected 12 items fit the hypothesised bifactor model with three unique factors and one general sensitivity factor (Lionetti et al., 2018). The bifactor model was then compared to a one-factor and a three-factor model, applying a qualitative evaluation of the fit indices of the models, as well as the CFI criterion, and the scaled $\chi^{2}$ difference tests (Satorra, 2000). If the difference in the CFIs between two nested models is smaller than $|0.01|$, the hypothesis of no difference in fit between the two competing models should not be rejected (Cheung \& Rensvold, 2002). For the evaluation of goodness of fit, we considered the following indices: the Tucker Lewis index (TLI), the Comparative Fit Index (CFI), the Root Mean Square Error of Approximation (RMSEA) and the Standardized Root Mean Square Residuals, SRMR). CFI and TLI values of > .95 and > .97 , respectively, were considered as acceptable and good fit. For RMSEA, values < .05 were considered as reflecting a good fit with values ranging from .05 and .08 indicating an adequate fit. For SRMR, values less than .08 were considered to reflect good fit (Schermelleh-Engel et al., 2003). For descriptive purposes, reliability indices including Cronbach's alpha, McDonald's (1999) omega and hierarchical omega were considered (reported in supplementary information). 
In order to explore the association between sensitivity and personality, we computed bivariate correlations between the HSP-12 scale (including the three subscales) and the Big Five personality traits (including each of their six facets). In addition, we ran a multiple regression model with all five personality dimensions simultaneously included as predictors of sensitivity in order to estimate how much of the variance of the HSP-12 total score is accounted for by established personality traits. Finally, we tested all correlations between HSP-12 and the personality scales that were higher than $r=.50$ for divergent validity (i.e., whether traits are distinguishable from each other) by considering the heterotrait-monotrait (HTMT) ratio of correlations in a multitrait-multimethod matrix. This approach includes computing the average of the correlations of items across constructs that measure different dimensions relative to the average of the correlations of items within the same construct. HTMT values equal or lower than .85 are considered to satisfy divergent validity (Henseler et al., 2015).

Analyses were run in R, using package lavaan (Rosseel, 2012) for Confirmatory Factor Analyses and package semTools (Pornprasertmanit et al., 2013) for computing the HSP scale reliability indices and estimating divergent validity.

\section{Results}

The mean value of the HSP-12 was $M=4.34, S D=.89$, which is comparable to the mean of the HSP-27 in our previous samples (Lionetti et al., 2018), and characterised by a normal distribution (see supplementary information). When comparing the HSP-12 and HSP-27 mean scores in the sample of our previous paper (Lionetti et al., 2018), the correlation between the two scales was very high, with $r=.94, p<.001$. The mean values of the subscales were $M=4.70(S D=1.18)$ for EOE, $M=4.42(S D=1.06)$ for AES, and $M=$ $3.64(S D=1.43)$ for LST. The subscales EOE and LST were strongly correlated with each 
other $(r=.56, p<.001)$ whereas the correlation was small between EOE and AES subscale $(r$ $=.08, p=.03)$ and moderate between LST and AES $(r=.24, p<.001)$.

According to the CFA, a one factor solution had a poor fit to the data (CFI $=.61$, TLI $=.52, \mathrm{RMSEA}=.16, \mathrm{SRMS}=.13$ ), while the hypothesised bifactor structure fit the data well, with CFI $=.95, \mathrm{TLI}=.92, \mathrm{RMSEA}=.06$, and SRMS $=.05$. The three-factor model had comparable fit indices $(\mathrm{CFI}=.94, \mathrm{TLI}=.92, \mathrm{RMSEA}=.04, \mathrm{SRMS}=.04)$, but the delta CFI criterion, equal to 0.013 , and the $\chi 2 \operatorname{DIFF}(9)=28.338, p<.001$, provided stronger support for the bifactor model. Internal consistency for the total scale was good with $\alpha=.78$ (further reliability indices are reported in the supplementary information).

According to bivariate correlations, the total score of HSP-12 was significantly associated with all Big Five personality dimensions except agreeableness $(r=.02, p=.53$. The most notable associations with the total sensitivity score emerged for neuroticism $(r=$ $.51, p<.001)$ and its facets anxiety $(r=.52, p<.01)$ and vulnerability $(r=.55, p<.001)$. Significant and large correlations were identified between the subscale EOE and the neuroticism facets of anxiety $(r=.67, p<.001)$, depression $(r=.50, p<.001)$, selfconsciousness $(r=.53, p<.001)$, and vulnerability $(r=.68, p<.001)$. The HSP-12 total score was also moderately and negatively correlated with extraversion $(r=-.30, p<.001)$, which seemed to be mostly due to the strong association with the subscale EOE $(r=-.46, p<$ $.001)$. The moderate correlation between HSP-12 and openness $(r=.25, p<.001)$ was largely the result of the high correlation with the subscale AES $(r=.63, p<.001)$. AES was positively associated with all openness facets but particularly strongly with Artistic Interests $(r=.64, p<.001)$. Finally, the association between HSP-12 and conscientiousness was negative and small $(r=-.15, p<.001)$. Bivariate correlations between all sensitivity and personality dimensions and facets are reported in Table 2. 
When all Big Five factors were included simultaneously in a multiple regression model as predictors of the HSP-12 total score, the five personality traits combined explained $36 \%$ of the variance of HSP-12 $(F(5,716)=82, p<.001)$. Consistent with bivariate correlations neuroticism was the strongest predictor $(\beta=.53, p<.001)$, followed by openness $(\beta=.32, p<.01)$, extraversion $(\beta=-.08, p=.03)$, conscientiousness $(\beta=.07, p=.05)$ and agreeableness $(\beta=-.01, p=.83)$. Divergent validity between HSP-12 and personality traits was explored in relation to the sensitivity total score and neuroticism, subscale EOE and neuroticism, as well as between subscale AES and openness, both at the factor and facet level (i.e., for all correlations with $r=.50$ or higher). HTMT ratio values for HSP-12 and neuroticism, and its facets anxiety and vulnerability were $.69, .67$, and .74 , respectively. Concerning EOE, HTMT ratio values for the correlations with neuroticism and its facets anxiety, depression, self-consciousness, and vulnerability were $.76, .75, .50, .67$, and .83 , respectively, supporting divergent validity between the subscale EOE and all neuroticism facets. Finally, for the relationship between subscale AES and openness, the HTMT ratio was .86 , and for AES and the openness facet artistic interest it was .93, suggesting that these (sub)scales do not capture independent constructs and can't be distinguished from each other.

--- Insert here Table 2 ---

\section{Discussion}

According to Study 1, the newly developed brief HSP-12 scale yielded a similar total mean score and distribution as the original 27-item scale, with total scores being highly correlated. Internal consistency was good for the total score. Based on Confirmatory Factor Analysis the 12-item HSP scale provided a good fit with the hypothesised bifactor model made up of three distinct subscales and a general sensitivity factor (the one factor and three 
factor solutions performed worse). Importantly, the fit of the 12-item HSP scale with a bifactor structure was better than the one reported with the original 27-item scale (Lionetti et al., 2018), suggesting that the newly developed HSP-12 represents a promising brief measure for environmental sensitivity with psychometric properties that are similar or better than those of the original 27-item scale.

Associations with the Big Five personality traits and facets provide a clear picture of how environmental sensitivity measured with the HSP-12 relates to common personality traits. Sensitivity correlated most strongly and positively with high neuroticism (this association was mostly due to the subscale EOE and to a lesser degree to subscale LST), followed by negative correlations with low extraversion and high openness (the positive association with openness was mostly due to correlations with the subscale AES). When considering all five personality traits simultaneously, neuroticism and openness emerged as the most relevant predictors with a much weaker role of extraversion which is consistent with a recent meta-analysis (Lionetti, Pastore, et al., 2019). The multiple regression model further suggests that the five personality factors in total account for only about a third of the variance in HSP-12. Investigation of highly correlated traits suggested that neuroticism and all its facets can be discriminated from the HSP-12 and its subscales despite the substantial degree of shared variance. However, the personality trait of openness, and in particular its facet Artistic Interest, could not be distinguished from the subscale AES whilst only moderately correlating with the HSP-12 total score. Consequently, the subscale AES seems to reflect the personality trait of Openness, at least when measured with the IPIP scale. In sum, findings suggest that sensitive individuals are characterised by a specific personality profile of both high neuroticism and high openness and to a lesser degree low extraversion and low conscientiousness. These associations are largely consistent with theories of environmental sensitivity, according to which general sensitivity is manifested in both increased reactivity to 
negative (i.e., neuroticism) as well as positive experiences (i.e., openness) (Aron et al., 2012; Ellis et al., 2011; Pluess, 2015). Importantly, although some of the sensitivity subscales are highly correlated with some facets of the Big Five personality traits, this is much less pronounced for the HSP-12 total score. Given that sensitivity appears to reflect the combination of the common personality dimensions of neuroticism and openness, it is therefore advisable to use the total score as an index of environmental sensitivity rather than considering the three subscales individually.

\section{Study 2}

Although sensitivity measured with the HSP scales generally reflects a continuous and normal distributed trait, it has originally been suggested that sensitivity should be considered categorical (e.g., Aron et al., 2012; Boyce \& Ellis, 2005) with people falling into two distinct sensitivity groups: A highly sensitive group (sometimes referred to as “Orchids”), estimated to make up about $20 \%$ of the population, and a low sensitive group (referred to as "Dandelions") who represent the remaining $80 \%$ of the population. However, when this theoretical and widely popular proposition was tested empirically with the application of Latent Class Analysis on all items from the HSP-27 scale in adults (Lionetti et al., 2018) as well as from the 12-item HSC scale in children and adolescents (Pluess et al., 2018), findings consistently suggested the existence of three rather than two groups: About $30 \%$ of the population fell into a highly sensitive orchids category, and another $30 \%$ into a low sensitive dandelions group, while the remaining $40 \%$ represented a medium sensitivity group, the "Tulips" category (Lionetti et al., 2018). The aim of Study 2 was first to investigate whether the same three sensitivity groups emerge with the HSP-12 scale when applying a Latent Class Analysis across all 12 items and then to identify exploratory cut-off scores for these three groups.

\section{Methods}


Participants. The sample from Study 1 was combined with an additional sample of 230 psychology undergraduate students from Queen Mary University of London in the UK. The mean age of the additional student sample was 22.3 years $(S D=5.5)$ and $69.0 \%$ of the participants were female. Ethnic distribution, based on $98.3 \%$ respondents providing information on ethnicity, was as follows: $42.6 \%$ Caucasian, 33.5\% Asian-Indian, $7.0 \%$ African and $15.2 \%$ mixed/other, reflecting the typical ethnic composition of the diverse undergraduate population at this university. The study was approved by the institutional ethics committee (Queen Mary University of London). The combined sample included $\underline{952}$ adults (mean age $=27.9, S D=6.8,68 \%$ female). The ethnic composition of the combined sample was: $66.3 \%$ white/Caucasian, $19.3 \%$ Asian-Indian, $4.4 \%$ African/Caribbean, and 9.2 $\%$ mixed/other.

Procedures and Measures. Participants completed the same version of the HSP-12 scale (see Study 1). All 12 individual HSP items were included in the Latent Class Analysis.

Data Analysis. In order to test the existence of different sensitivity groups we performed a series of Latent Class Analyses (LCA) on all 12 HSP items (the total score or subscale structure were not considered). In order to identify the optimal number of latent classes, we considered the following set of criteria: 1) Akaike's Information Criterion (AIC), 2) Bayesian Information Criterion (BIC), 3) Lo-Mendell-Rubin adjusted likelihood ratio test (LMR-A), and 4) Entropy. AIC and BIC are comparative indices, with lower values indicating a better fit to the data. The LMR-A index compares the fit of the specified class solution to a model with one fewer class and provides a $p$-value which indicates whether the specified model provides a significantly better fit to the data than the more parsimonious model with one fewer class. Finally, entropy refers to the confidence with which individuals can be categorised into the number of tested classes with values closer to 1 being indicative of a clearer delineation of class membership (Nylund et al., 2007). Cut-off scores between 
groups were determined by considering the intersection points of the distribution of detected classes. Latent Class Analysis was performed using Mplus version 7 (Muthén \& Muthén, 1998-2015).

\section{Results}

The Latent Class Analysis supported the hypothesised three-class solution which fitted the data best with a significant LMR-A $(p=.02)$, adequate entropy (.77) and lower BIC and AIC compared to the two-class model (results are reported in Table 3). Tested models with one, two, four, five, and six class models had to be rejected given that none of them fitted the data significantly better than the three-class model. According to the three class solution, $23 \%$ of participants $(n=219)$ belonged to the low sensitive group (HSP-12 $M=$ 3.37, $S D=.60 ;$ EOE $M=2.98, S D=.62 ;$ AES $M=4.50, S D=1.10 ;$ LST $M=2.54, S D=$ $1.11), 40 \%(n=383)$ to the medium sensitive group (HSP-12 $M=4.10, S D=.47$; EOE $M=$ $4.64, S D=.61 ;$ AES $M=4.17, S D=1.04 ;$ LST $M=3.09, S D=.96)$ and $37 \%(n=350)$ to the high sensitive group (HSP-12 $M=5.21, S D=.45$; EOE $M=5.64, S D=.64$; AES $M=4.87$, $S D=.93 ;$ LST $M=4.96, S D=.93$ (see Figure 1 in supplementary material). According to the distribution of the three classes (see Figure 2), the intersection between the curves resulted in suggested cut-offs of 3.87 between low and medium groups and 4.63 between the medium and high groups.

$$
\begin{aligned}
& \text {--- Insert Figure } 2 \text { here--- } \\
& \text {--- Insert Table } 3 \text { here--- }
\end{aligned}
$$

\section{Discussion}

The results of the Latent Class Analysis confirmed the hypothesised existence of the same three sensitivity groups as previously identified in multiple independent samples of children, adolescents using the HSC scale (Pluess et al., 2018) and adults with the HSP-27 scale (Lionetti et al., 2018). However, in the current sample and with the HSP-12 scale the 
proportion of low sensitive adults was slightly lower (23\% versus $30 \%$ ) and the percentage of highly sensitive individuals was a bit higher (37\% versus $30 \%$ ) while the medium group making up $40 \%$ of the sample was similar to previous studies. Across the three groups, the total score as well as the EOE and LST subscales followed a low-to-medium-to-high trend except for the subscale AES which was highest in the high sensitive individuals but did not differentiate well between the low and medium groups. Important to mention, according to the distribution of the three groups, there was substantial overlap between the three groups, especially between the medium and low sensitive groups. Consequently, the cut-offs derived from the intersection points should be considered exploratory and applied with caution. As suggested elsewhere, sensitivity measured with the HSP scales should be considered a continuous dimension along which people appear to fall into three different groups (Lionetti et al., 2018; Pluess et al., 2018). Hence, it is advisable to use the continuous total score of the HSP-12 scale in research studies on sensitivity (and investigate extreme groups of the total score by comparing top and bottom $30 \%$ of the sample in follow-up analyses) rather than applying the exploratory cut-off scores in independent samples. However, the preliminary cut-off scores might be more helpful in applied settings in order to get a sense whether an individual is more likely falling into the low, medium, or high sensitivity group.

\section{Study 3}

Studies 1 and 2 provide important information on the psychometric properties of the HSP-12 scale, its relationship with established personality traits and whether sensitivity should be considered a continuous or rather a categorical trait. However, it remains to be tested whether environmental sensitivity measured with the HSP-12 does indeed predict whether people are more affected by the quality of their environment as predicted by theory (Pluess, 2015). Study 3 aimed to test whether sensitivity predicts the response to negative experiences applying a single-group experimental design in an authentic real-life context by 
investigating the effect of first-time teaching on the well-being of unexperienced teachers with limited training who often find their first year of teaching challenging (indicated by high numbers of drop out) (Kelchtermans, 2017). We recruited a sample of recent university graduates who enrolled into a well-respected teacher training programme provided by a large teaching charity in the United Kingdom. The programme aims to train talented graduates from leading universities in just a few weeks over summer before getting them to teach fulltime in schools attended by students from predominately disadvantaged backgrounds. During the following two years trainees receive further training whilst teaching full-time before they graduate with a university-level teaching qualification. Although trainees are supported by experienced mentors throughout the course, the first few months tend to be particularly stressful when trainees are thrown into the deep end of teaching without having much experience yet. We assessed sensitivity with the HSP-12 scale during the summer training and tracked the development of their self-reported well-being across the following 10 months during their first year of teaching. Informed by theories of environmental sensitivity, we hypothesised that sensitivity would predict the trajectory of well-being during the first year of teaching with more sensitive teacher trainees showing a stronger decline in well-being compared to low sensitive individuals in response to the challenge of first-time teaching.

\section{Methods}

Participants. We recruited 110 new teacher trainees from a leading teaching charity in the United Kingdom. The age range of participants was between 21-35 years $(M=23.53$, $S D=2.33)$ and the majority of participants were female (78\%). Ethnic distribution was as follows: $84.5 \%$ Caucasian, $6.4 \%$ Arab/Persian, $4.6 \%$ Asian-Indian and 4.6\% other ethnic backgrounds. The study was approved by the institutional ethics committee (Queen Mary University of London). 
Procedure. All data for this longitudinal single-group experimental study was collected online. Teacher trainees were initially invited per email to participate in the study. Interested trainees then completed the baseline assessment several weeks before they started teaching their first class (after providing informed consent). Baseline assessment included demographic information, the HSP-12 scale, and a measure of self-reported broad well-being, as well as a range of other measures not included in this analysis (i.e., perceived stress, psychological distress, mood, subjective vitality, self-esteem, self-efficacy, depression, Big Five personality, religiosity, coping behaviour, and social support). Participants who completed the baseline assessment were then contacted every other month across a period of ten months (follow-up 1 to 5) in order to complete short follow-up assessments which included the same broad wellbeing measure that was used at baseline in addition to other measures not included in this analysis (i.e., perceived stress, psychological distress, mood, and subjective vitality). The majority of the sample (i.e., 77\%) provided information on at least four of the six separate assessments. The remaining $23 \%$ completed surveys only for one or two assessments. However, all 110 participants were included in the analysis given that the applied Latent Growth Curve modelling accounts for cases with missing data.

Measures. Environmental sensitivity was measured with the newly developed HSP12 (see Study 1). Internal consistency in the current sample was good with alpha $=.80$. Selfreported well-being was assessed with the Mental Health Continuum Short Form (MHC-SF) (Keyes, 2002). The MHC-SF includes 14 items that describe positives states (e.g., "satisfied with life", "life has a sense of direction or meaning to it", "interested in life") which are rated by participants on a 6-point rating scale regarding their frequency of experience, from 1 $=$ "never" to $6=$ "everyday". In the current sample, we used the total score with higher values reflecting higher well-being. Cronbach's alpha was good with alpha $=.88$. 
Data Analysis. After considering unadjusted associations between all included variables, the change in wellbeing across time was investigated with a series of Latent Growth Curve models (i.e., modelling linear versus quadratic slopes). We then tested whether environmental sensitivity influenced the degree of change in self-reported well-being across the six assessments (i.e., baseline and follow-up one to five). Significant effects were investigated further with simples slopes of extreme HSP-12 groups defined as the top and bottom $30 \%$ of the sample. Latent growth curve models were run in programme $\mathrm{R}$ using the lavaan package (Rosseel, 2012).

\section{Results}

Bivariate correlations between gender, age, HSP-12, and well-being across the six assessments are reported in Table 4. Importantly, HSP-12 was not significantly associated with well-being at any time point except the second follow-up assessment $(r=-.37, p<.01)$.

\section{--- Insert Table 4 here ---}

According to Latent Growth Curve modelling the model with a quadratic slope fitted the data reasonably well $(\mathrm{CFI}=.92, \mathrm{TLI}=.93, \mathrm{SRMR}=.09$, parameters are reported in the appendix $)$ and significantly better than a linear change model $(\chi 2[\mathrm{DIFF}](4)=18.5, p<.01)$. When the HSP-12 total score was included as predictor of intercept and slope of well-being, controlling for gender, it significantly predicted the quadratic slope $(\beta=.50, p=.03)$ but not the intercept at baseline $(\beta=-.04, p=.83)$. According to additional analyses with the intercept centred at each of the follow-up assessments, HSP-12 was significantly associated with a decrease in wellbeing at follow-up one, two, and three with $\beta=-.24(p=.08), \beta=-.31$ $(p=.02)$, and $\beta=-.29(p=.03)$ respectively, and with a subsequent gradual increase with $\beta=$ $-.20(p=.12)$, and $\beta=-.02(p=.92)$ at follow-up four and five. 
According to plotted slopes of extreme groups (see Figure 3), there was no significant change in well-being for teacher trainees characterised by low sensitivity (i.e., bottom $30 \%$ of HPS-12, quadratic term was $\beta=.67, p=.30$ ). On the contrary, highly sensitive trainees (i.e., top $30 \%$ of HSP-12,) showed a clear decline in well-being once teaching started with a full recovery towards the last follow-up (quadratic term was $\beta=1.78, p=.002$ ).

--- Insert here Figure 3 ---

\section{Discussion}

Testing the environmental sensitivity hypothesis that more sensitive individuals would be more strongly and more negatively affected by the exposure to a potentially stressful environment (Pluess, 2015), we investigated whether sensitivity measured with the HSP-12 scale predicted a decline in well-being in teacher trainees during their first year of teaching. The study featured a unique natural single-group experiment with unexperienced first time teachers who often tend to experience their first year of teaching as especially challenging (Chaplain, 2008). Consistent with this hypothesis, HSP-12 emerged as a significant predictor of change in well-being over the period of 10 months, with more sensitive teacher trainees showing a significant decline in well-being followed by full recovery whereas low sensitive teachers appeared unaffected by the same putative stressful experience. Importantly, the study was conducted in an authentic real-life context with high external validity rather than under highly controlled and artificial lab conditions. Hence, findings provide valid empirical evidence that the HSP-12 scale measures individual differences in environmental sensitivity. Importantly, heightened sensitivity was not associated with well-being at baseline (or later follow-up assessment) but with decreased well-being scores shortly after the school year started. This suggests that HSP-12 does not simply reflect generally lower well-being but rather increased sensitivity to the particular quality of the environment. 


\section{Study 4}

According to environmental sensitivity theories, sensitive individuals are not only more vulnerable to the negative effects of adverse exposures but also more likely to benefit from the positive effects of supportive experiences (Aron et al., 2012; Belsky et al., 2007; Ellis et al., 2011; Pluess, 2015). While Study 3 provided evidence that HSP-12 indeed predicts a stronger negative response to an adverse experience, Study 4 aimed to investigate whether sensitive individuals are also more affected by the positive effects of a positive influence. In order to do so we tested the moderating effect of HSP-12 in a lab-based mood induction experiment. After participants completed the HSP-12 scale, they rated their positive mood shortly before and directly after watching a video clip with uplifting content. We hypothesised that sensitivity would significantly influence the pre-post change in positive mood with more sensitive individuals showing a stronger increase than less sensitive ones.

\section{Methods}

Participants. The study included 80 psychology undergraduate students from a university in the United Kingdom who participated in the study in exchange for course credit during the first year of their course. Students ranged in age between 18-26 years $(M=18.99$, $S D=1.41)$ and the majority $(89 \%)$ was female. Information on the ethnicity of the students was not collected and therefore not available. The study was approved by the institutional ethics committee (Queen Mary University of London).

Procedure. Study participation was offered to first year psychology students in order to obtain required course credits for active involvement in research. Interested participants were invited to the lab and, after providing informed consent, asked to complete a range of tasks, which included a mood induction experiment (the other tasks focused on the measurement of creativity pre and post mood induction and are not discussed further given that they are not relevant for the current study). Participants completed the HSP-12 scale during the baseline 
assessment at the beginning of the session. The positive mood induction featured a threeminute video in which the camera followed a group of young people in the USA handing out gifts to homeless people who consequently respond with gratitude (https://www.youtube.com/watch?v=GoGtaXt5f1w). Participants were asked to rate their positive mood on a visual analogue scale before and directly after watching the video. Importantly, they did not know what to expect in the video (participants were debriefed about the goals of the study after completion of the session).

Measures. Sensitivity was assessed with the HSP-12 scale (see Study 1). Positive mood was measured with the help of a visual analogue scale, a $10 \mathrm{~cm}$ long drawn line labelled "Not Happy at all" at the left extreme and "Very Happy" at the right extreme. Participants were asked to mark the line with a pen somewhere between the extremes in order to indicate how they felt momentarily. The distance from the left end to the mark was measured in mm in order to obtain a measure of positive mood.

Data Analysis. After considering unadjusted correlations between all variables, we applied a series of mixed-effects regression models with random intercepts predicting mood. The first model included only gender, the second model gender and time (i.e., assessment before and after the video), the third model gender, time and sensitivity (i.e., HSP-12). The final model included gender, time, and HSP-12 as main effects as well as the interaction between time and HSP-12. All four models were compared regarding their fit using the likelihood-ratio test and Akaiake weights, representing the probability of each model being the best one against the set of models considered (Wagenmakers \& Farrell, 2004). In order to follow up significant interactions, we computed simple slopes for extreme groups (i.e., top and bottom $30 \%$ of the HSP-12) between pre and post scores. Analyses were conducted in Programme R using package lme4 for mixed models (Bates et al., 2007).

\section{Results}


Bivariate correlations between study variables are reported in Table 5. Female gender was significantly correlated with higher positive mood after the video. Importantly, HSP-12 was not correlated with positive mood neither before nor after the video-clip.

$$
\text { --- Insert Table } 5 \text { here--- }
$$

According to the comparison of mixed-effects regression models, the second model which included gender and time performed significantly better than model 1 which included only gender (see Table 6). The inclusion of HSP-12 in model 3 did not lead to a further improvement. However, the addition of the interaction term between HSP-12 and time in model 4 led to a significantly better fit compared to all other models as supported by Akaike weights. The regression parameter for the interaction term between time and the HSP-12 was significant with $\beta=.63(p<.01)$. In order to follow up the significant interaction, we computed and plotted simple slopes for high and low sensitive individuals (i.e., top and bottom $30 \%$ of HSP-12; see Figure 3). The slope between pre- and post-ratings of positive mood was higher in the high sensitive group with $\beta=.59(p<.01)$ compared to the low sensitive group with $\beta=.39(p=.01)$.

--- Insert Table 6 here ---

--- Insert Figure 4 here ---

\section{Discussion}

Study 4 provides empirical evidence from a mood induction experiment suggesting that more sensitive individuals are indeed more responsive to a positive influence compared to less sensitive people. According to mixed-effects regression models sensitivity measured with the HSP-12 significantly moderated the short-term effects of the positive mood induction with a video clip on pre-post changes in positive mood. Follow-up analyses showed that although both low and high sensitive individuals showed a significant increase in 
positive mood after watching the video, the change was more pronounced in the high sensitive group. These findings provide important experimental evidence that the HSP-12 does not only capture heightened sensitivity to negative but also to positive experiences. Furthermore, similar to Study 3, HSP-12 was not associated with the outcome variable before the exposure, suggesting that HSP-12 does not necessarily capture stable differences in emotional disposition but predicts the response to environmental influences.

\section{General Discussion}

The current study aimed at describing the integrative framework of environmental sensitivity (Pluess, 2015) and providing new evidence for individual differences in sensitivity, including the development of a short version of the Highly Sensitive Person scale (Aron \& Aron, 1997) in order to create a reliable, robust and valid but brief measure of environmental sensitivity for adults. Given reported links between sensitivity and common personality traits (Lionetti, Pastore, et al., 2019), we then investigated associations between sensitivity and the Big Five personality dimensions and underlying facets, followed by testing whether the distribution of sensitivity in our sample fits the same three group solution that emerged in previous work (Lionetti et al., 2018; Pluess et al., 2018). Finally, we tested teacher trainee's adjustment to the challenge of first time teaching as well as the response of study participants to a positive mood induction in order to experimentally test and validate whether the new sensitivity scale predicts the hypothesised heightened responsivity to both negative and positive exposures in more sensitive individuals.

\section{General Interpretation of Empirical Findings}

According to Study 1 the newly developed 12-item Highly Sensitive Person scale (HSP-12) reflects the same bifactor structure as detected in the original 27-item scale but with improved fit. The included items all load both on a general sensitivity factor as well as on the three individual components of sensitivity originally reported by Smolewska et al. (2006): 
Ease of Excitation, Low Sensory Threshold, and Aesthetic Sensitivity. Importantly, the HSP12 is characterised by good psychometric properties and correlates highly with the original 27-item scale. Consequently, we conclude that the HSP-12 is a reliable measure of sensitivity with similar or better psychometric properties than the original scale. Having access to a shorter yet reliable measure of sensitivity will make it easier for researchers to consider this construct for inclusion in future studies. Study 1 also provided valuable insights regarding the relationship between sensitivity and the Big Five personality traits and facets. Sensitivity measured with the HSP-12 was associated with all of the five personality traits except agreeableness. However, when all five traits were included simultaneously in the same regression model, only neuroticism and openness to experiences emerged as predictors of sensitivity with moderate to strong effects, which is consistent with previous work (Bröhl et al., 2020; Lionetti, Pastore, et al., 2019). Furthermore, all five personality dimensions combined accounted for about a third of the variance in sensitivity which suggests that although associated, sensitivity is not completely explained by established personality traits. The detected associations between sensitivity and the Big Five personality facets further elucidate how personality relates to sensitivity with facets of neuroticism and openness to experiences highly correlating with the Ease of Excitation and Aesthetic Sensitivity subscales, respectively. Importantly, divergent validity was supported for all associations except for the correlation between the artistic interest facet of openness to experiences and the aesthetic sensitivity component of the HSP-12 which were not distinguishable from each other. These findings suggest that the total score of the HSP-12 should be used as an index of sensitivity — rather than the three individual subscales — given that the total score can be more reliably differentiated from all dimensions and facets of the Big Five personality traits.

Study 2 confirmed previous findings featuring other sensitivity scales according to which individuals seem to fall into one of three classes along a continuum from low, to 
medium, to high sensitivity (Lionetti et al., 2018; Pluess et al., 2018). Whilst this empirically confirmed categorical perspective on sensitivity might be helpful when assessing an individual's sensitivity in more applied settings — with the help of the estimated cut-off scores - the normal distribution of the total score points towards the existence of a continuous sensitivity spectrum. Given that it is not completely clear yet whether sensitivity is better conceptualised as a continuous or categorical measure and in light of the risk that cut-offs may misplace some individuals (Preacher et al., 2005), we recommend using the continuous score (and following-up interactions by plotting simple slopes for top and bottom 30\%) when conducting research that includes the trait of sensitivity measured with the HSP-12.

The results of Study 3 and 4 add new experimental evidence to the field that people differ significantly in their response to both negative and positive experiences. More specifically, these studies provide important empirical evidence that the HSP-12 scale reflects a valid assessment of environmental sensitivity given that it predicted the hypothesised heightened response to both negative and positive experiences in experimental settings. According to Study 3, sensitivity was associated with individual differences in teacher trainee's adjustment to the challenge of first time teaching with more sensitive teachers showing a drop in well-being followed by complete recovery whereas the well-being of less sensitive teachers remained unchanged. Importantly, the response to the stressful experience of first time teaching was assessed longitudinally with sensitivity measured before the actual exposure and well-being tracked across several months during the exposure. Results provide clear evidence that more sensitive individuals are more strongly affected by stressful experiences, but also show that they don't necessarily have lower well-being before the exposure and that they manage to recover over time, which may help to differentiate sensitivity from related traits such as neuroticism. Study 4 featured a lab-based experiment on positive mood induction and confirmed the hypothesis that sensitivity measured with the 
HSP-12 would also predict a stronger positive response to a positive experience. Although all participants included in the study seemed to show increased happiness after watching a short video with positive content, the more sensitive people were, the more they benefited from the positive effects of this positive exposure. Taken together, results from Study 3 and Study 4, strengthen the framework of environmental sensitivity more generally, and provide important empirical support for the HSP-12 scale as a valid measure of sensitivity to the environment, reflected in both heightened vulnerability to the negative effects of a stressful condition (Study 3) as well as increased propensity to benefit from positive experiences (Study 4). In contrast, less sensitive individuals proved to be more resilient to stress but also less responsive to the positive effects of the positive exposure.

\section{Strengths and Limitations of Presented Empirical Work}

The research reported in this paper is characterised by several strengths such as the application of a broad range of research methodology, statistical approaches, and study designs from large cross-sectional surveys to lab-based experiments across four individual studies with three independent samples. More specifically, strengths included: 1) a large sample of $N=1,104$ across all studies, 2) the investigation of the association between sensitivity and the Big Five personality dimensions at the facet level (Study 1), 3) testing individual differences in sensitivity to a stressful experience in an authentic real-life context with longitudinal assessment from before until the end of the exposure (Study 3), 4) overcoming limitations of correlational research by testing individual differences in sensitivity to positive exposures in a lab-based experiment (Study 4). However, the findings must also be considered in light of some methodological limitations. Most importantly, all data were based exclusively on self-report and collected from participants living in the United Kingdom. Furthermore, the samples of the experimental studies were relatively small. Hence, 
the reported findings remain to be confirmed and replicated in larger samples across different populations and in studies featuring more objective measures and procedures.

\section{Implications of Theory and Empirical Evidence}

The findings of this multi-study paper confirm several aspects of the integrated perspective of environmental sensitivity. Most importantly, results show that people differ in their sensitivity to environmental influences and that sensitivity can be measured with a short self-report scale. Although measured sensitivity reflects a specific personality profile of both heightened neuroticism and openness to experiences (including at the facet level), differences in sensitivity are not completely explained by the common Big Five personality traits. Furthermore, sensitivity can be conceptualised both as continuous and categorical. Similarly to most other common human traits, sensitivity is normally distributed in the population. However, according to the applied data-driven statistical approach people can also be categorised along this continuum into low, medium and high in sensitivity. This suggests that there are qualitative differences between these three groups which need to be investigated further (e.g., see Lionetti et al., 2018). Going beyond correlational and cross-sectional designs, the current study also provides important experimental evidence that people that score high in sensitivity are indeed more affected by negative and/or positive environmental influences. This means that sensitivity should be considered in the field of personality psychology as an additional and relatively distinct human trait that describes fundamental human behaviour and explains some of the widely observable differences in people's responsivity or reactivity to various experiences. An important practical implication of the theory and reported findings is that just as not all people will respond similarly to adversity, they also won't benefit to the same degree from positive exposures. Hence, inter-individual variability in response to environmental influences should be considered the norm rather than an exception and a distinct differential and quantifiable feature of human nature. 


\section{Future Directions}

Given that research on environmental sensitivity is still in a relatively early stage compared to other common human traits, various questions remain to be addressed in future work. This includes the continued improvement of sensitivity measures that reach beyond self-report by investigating and identifying the most relevant genetic, physiological, neuroscientific, cognitive, and behavioural markers of sensitivity. In addition, future work should explore the specific biological and psychological mechanisms underlying environmental sensitivity and also investigate the development of sensitivity over time (from the early prenatal period onwards) in order to understand its stability across the life course and investigate how an individual's level and type of sensitivity is shaped by the environment as well as the interplay with genetic factors. Finally, future research should continue to investigate the moderating function of sensitivity in relation to various environmental influences (including the work environment) and across different populations, as well as further explore the detected associations with established personality traits.

\section{Conclusion}

To summarise, the series of studies included in this paper confirm several aspects of the proposed integrated theory of environmental sensitivity and provide new evidence that individual differences in sensitivity to the environment can be measured reliably in adults with a short self-report measure and predict the response to both negative and positive environmental influences. Furthermore, results confirm that although sensitivity measured with a short scale reflects a continuum from low to high, people seem to fall into three sensitivity groups along this continuum—dandelions, tulips, and orchids. Finally, our study provides evidence that sensitivity is reflected in a specific personality profile that is characterised by heightened neuroticism (and most of its facets) as well as heightened openness to experiences (and several of its facets). In conclusion, adults differ substantially in 
their sensitivity to environmental influences and such differences in trait sensitivity can be measured reliably with a short and simple, yet psychometrically robust and empirically validated self-report measure. 


\section{Acknowledgements}

We thank Saajida Ahmad, Channon Allen-Gulley, Madeleine King, Annabelle Tiam-Fook, and Aiysha Zahid for their contribution to data collection at Queen Mary University of London. We also thank Teach First and their teacher trainees for their supporting role in the research. Finally, we thank all the participants that generously contributed to the various studies report here. Francesca Lionetti was supported with a grant of the European Commission H2020 -MSCA-IF-2015-704283. 


\section{References}

Acevedo, B. P., Aron, E. N., Aron, A., Sangster, M. D., Collins, N., \& Brown, L. L. (2014). The highly sensitive brain: an fMRI study of sensory processing sensitivity and response to others' emotions. Brain and behavior, 4(4), 580-594.

Aron, E. N., \& Aron, A. (1997, Aug). Sensory-processing sensitivity and its relation to introversion and emotionality. Journal of Personality and Social Psychology, 73(2), 345-368. https://www.ncbi.nlm.nih.gov/pubmed/9248053

Aron, E. N., Aron, A., \& Jagiellowicz, J. (2012, Aug). Sensory processing sensitivity: a review in the light of the evolution of biological responsivity. Personality and Social Psychology Review, 16(3), 262-282. https://doi.org/10.1177/1088868311434213

Assary, E., Zavos, H. M. S., Krapohl, E., Keers, R., \& Pluess, M. (2020, Jun 3). Genetic architecture of Environmental Sensitivity reflects multiple heritable components: a twin study with adolescents. Molecular Psychiatry. https://doi.org/10.1038/s41380$\underline{020-0783-8}$

Bakermans-Kranenburg, M. J., \& van IJzendoorn, M. H. (2011, Feb). Differential susceptibility to rearing environment depending on dopamine-related genes: new evidence and a meta-analysis. Development and Psychopathology, 23(1), 39-52. https://doi.org/10.1017/s0954579410000635 
Bates, D., Sarkar, D., Bates, M. D., \& Matrix, L. (2007). The lme4 package. $R$ package version, 2(1), 74 .

Belsky, J. (1997). Variation in susceptibility to rearing influences: An evolutionary argument. Psychological Inquiry, 8, 182-186.

Belsky, J., Bakermans-Kranenburg, M. J., \& van IJzendoorn, M. H. (2007, Dec). For better and for worse: Differential susceptibility to environmental influences. Current Directions in Psychological Science, 16(6), 300-304. https://doi.org/DOI 10.1111/j.1467-8721.2007.00525.x

Belsky, J., Jonassaint, C., Pluess, M., Stanton, M., Brummett, B., \& Williams, R. (2009, Aug). Vulnerability genes or plasticity genes? Molecular Psychiatry, 14(8), 746-754. https://doi.org/10.1038/mp.2009.44

Belsky, J., \& Pluess, M. (2009, Nov). Beyond diathesis stress: differential susceptibility to environmental influences. Psychological Bulletin, 135(6), 885-908. https://doi.org/10.1037/a0017376

Belsky, J., \& Pluess, M. (2011). Beyond Adversity, Vulnerability and Resilience: Individual Differences in Developmental Plasticity. In D. Cicchetti \& G. I. Roisman (Eds.), Minnesota Symposium on Child Psychology, Vol.36: The Origins and Organization of Adaptation and Maladaptation (pp. 379-422). John Wiley. 
Belsky, J., \& Pluess, M. (2013, Nov). Beyond risk, resilience, and dysregulation: phenotypic plasticity and human development. Development and Psychopathology, 25(4 Pt 2), 1243-1261. https://doi.org/10.1017/S095457941300059X

Belsky, J., \& Pluess, M. (2016). Differential Susceptibility to Environmental Influences. In D. Cicchetti (Ed.), Developmental Psychopathology (3rd ed., Vol. 3, pp. 59). Wiley.

Boyce, W. T. (1996). Biobehavioral reactivity and injuries in children and adolescents. In M. H. Bornstein \& J. Genevro (Eds.), Child development and behavioral pediatrics: Toward understanding children and health (pp. 35-58). Erlbaum.

Boyce, W. T., \& Ellis, B. J. (2005, Spring). Biological sensitivity to context: I. An evolutionary-developmental theory of the origins and functions of stress reactivity. Development and Psychopathology, 17(2), 271-301. https://www.ncbi.nlm.nih.gov/pubmed/16761546

Bröhl, A. S., Van Leeuwen, K., Pluess, M., De Fruyt, F., Bastin, M., Weyn, S., Goossens, L., \& Bijttebier, P. (2020, 2020/08/20). First look at the five-factor model personality facet associations with sensory processing sensitivity. Current Psychology. https://doi.org/10.1007/s12144-020-00998-5 
Chaplain, R. P. (2008, 2008/03/01). Stress and psychological distress among trainee secondary teachers in England. Educational Psychology, 28(2), 195-209. https://doi.org/10.1080/01443410701491858

Cheung, G. W., \& Rensvold, R. B. (2002, 2002/04/01). Evaluating Goodness-of-Fit Indexes for Testing Measurement Invariance. Structural Equation Modeling: A Multidisciplinary Journal, 9(2), 233-255. https://doi.org/10.1207/S15328007SEM0902_5

Drury, S. S., Gleason, M. M., Theall, K. P., Smyke, A. T., Nelson, C. A., Fox, N. A., \& Zeanah, C. H. (2012, Jul 16). Genetic sensitivity to the caregiving context: The influence of 5httlpr and BDNF val66met on indiscriminate social behavior. Physiology and Behavior, 106(5), 728-735. https://doi.org/10.1016/j.physbeh.2011.11.014

Duncan, L. E., \& Keller, M. C. (2011, Oct). A Critical Review of the First 10 Years of Candidate Gene-by-Environment Interaction Research in Psychiatry. American Journal of Psychiatry, 168(10), 1041-1049. https://doi.org/10.1176/appi.ajp.2011.11020191

Ellis, B. J., Boyce, W. T., Belsky, J., Bakermans-Kranenburg, M. J., \& Van IJzendoorn, M. H. (2011, Feb). Differential susceptibility to the environment: An evolutionaryneurodevelopmental theory. Development and Psychopathology, 23(01), 7-28. https://doi.org/10.1017/S0954579410000611 
Gard, A. M., Shaw, D. S., Forbes, E. E., \& Hyde, L. W. (2018, Dec). Amygdala Reactivity as a Marker of Differential Susceptibility to Socioeconomic Resources During Early Adulthood. Developmental Psychology, 54(12), 2341-2355. https://doi.org/10.1037/dev0000600

Goldberg, L. R. (1999). A broad-bandwidth, public-domain, personality inventory measuring the lower-level facets of several five-factor models. Personality Psychology Europe, 7, 7-28.

Gray, J. A. (1981). A critique of Eysenck's theory of personality. In H. J. Eysenck (Ed.), A model for personality (pp. 246-276). Springer-Verlag.

Gray, J. A. (1982). The neuropsychology of anxiety: An enquiry into thefunctions of the septo-hippocampal system. Oxford University Press.

Greven, C. U., Lionetti, F., Booth, C., Aron, E. N., Fox, E., Schendan, H. E., Pluess, M., Bruining, H., Acevedo, B., Bijttebier, P., \& Homberg, J. (2019, Mar). Sensory Processing Sensitivity in the context of Environmental Sensitivity: A critical review and development of research agenda. Neuroscience and Biobehavioral Reviews, 98, 287-305. https://doi.org/10.1016/j.neubiorev.2019.01.009 
Hartman, S., Freeman, S. M., Bales, K. L., \& Belsky, J. (2018). Prenatal stress as a risk-and an opportunity_factor. Psychological Science, 29(4), 572-580.

Henseler, J., Ringle, C. M., \& Sarstedt, M. (2015). A new criterion for assessing discriminant validity in variance-based structural equation modeling. Journal of the Academy of Marketing Science, 43(1), 115-135. https://doi.org/10.1007/s11747-014-0403-8

Johnson, J. A. (2014). Measuring thirty facets of the Five Factor Model with a 120-item public domain inventory: Development of the IPIP-NEO-120. Journal of Research in Personality, 51, 78-89.

Kagan, J. (1997). Temperament and the reactions to unfamiliarity. Child Development, 68(1), $139-143$.

Kagan, J., Reznick, J. S., \& Snidman, N. (1987, Dec). The physiology and psychology of behavioral inhibition in children. Child Development, 58(6), 1459-1473. http://www.ncbi.nlm.nih.gov/pubmed/3691195

Keers, R., Coleman, J. R., Lester, K. J., Roberts, S., Breen, G., Thastum, M., Bögels, S., Schneider, S., Heiervang, E., \& Meiser-Stedman, R. (2016). A Genome-Wide Test of the Differential Susceptibility Hypothesis Reveals a Genetic Predictor of Differential Response to Psychological Treatments for Child Anxiety Disorders. Psychotherapy and Psychosomatics, 85(3), 146-158. 
Keers, R., \& Pluess, M. (2017). Childhood quality influences genetic sensitivity to environmental influences across adulthood: A life-course Gene $\times$ Environment interaction study. Development and Psychopathology, 29(5), 1921-1933. https://doi.org/10.1017/S0954579417001493

Kelchtermans, G. (2017, 2017/11/17). 'Should I stay or should I go?': unpacking teacher attrition/retention as an educational issue. Teachers and Teaching, 23(8), 961-977. https://doi.org/10.1080/13540602.2017.1379793

Keyes, C. L. (2002). The mental health continuum: From languishing to flourishing in life. Journal of Health and Social Behavior, 207-222.

Kumsta, R., Stevens, S., Brookes, K., Schlotz, W., Castle, J., Beckett, C., Kreppner, J., Rutter, M., \& Sonuga-Barke, E. (2010, Jul). 5HTT genotype moderates the influence of early institutional deprivation on emotional problems in adolescence: evidence from the English and Romanian Adoptee (ERA) study. Journal of Child Psychology and Psychiatry and Allied Disciplines, 51(7), 755-762. https://doi.org/10.1111/j.1469$\underline{7610.2010 .02249 . \mathrm{x}}$

Lionetti, F., Aron, A., Aron, E. N., Burns, G. L., Jagiellowicz, J., \& Pluess, M. (2018). Dandelions, tulips and orchids: evidence for the existence of low-sensitive, mediumsensitive and high-sensitive individuals. Translational psychiatry, 8(1), 24. https://doi.org/10.1038/s41398-017-0090-6 
Lionetti, F., Aron, E. N., Aron, A., Klein, D. N., \& Pluess, M. (2019). Observer-rated environmental sensitivity moderates children's response to parenting quality in early childhood. Developmental Psychology.

Lionetti, F., Pastore, M., Moscardino, U., Nocentini, A., Pluess, K., \& Pluess, M. (2019, Aug). Sensory Processing Sensitivity and its association with personality traits and affect: A meta-analysis. Journal of Research in Personality, 81, 138-152. https://doi.org/10.1016/j.jrp.2019.05.013

Lo, M.-T., Hinds, D. A., Tung, J. Y., Franz, C., Fan, C.-C., Wang, Y., Smeland, O. B., Schork, A., Holland, D., \& Kauppi, K. (2016). Genome-wide analyses for personality traits identify six genomic loci and show correlations with psychiatric disorders. Nature Genetics.

[Record \#2167 is using a reference type undefined in this output style.]

Monroe, S. M., \& Simons, A. D. (1991, Nov). Diathesis-stress theories in the context of life stress research: implications for the depressive disorders. Psychological Bulletin, 110(3), 406-425. https://www.ncbi.nlm.nih.gov/pubmed/1758917

Muthén, L. K., \& Muthén, B. O. (1998-2015). Mplus User's Guide. Seventh Edition. Los Angeles, CA: Muthén \& Muthén. 
Nagel, M., Jansen, P. R., Stringer, S., Watanabe, K., de Leeuw, C. A., Bryois, J., Savage, J. E., Hammerschlag, A. R., Skene, N. G., \& Muñoz-Manchado, A. B. (2018). Metaanalysis of genome-wide association studies for neuroticism in 449,484 individuals identifies novel genetic loci and pathways. Nature Genetics, 50(7), 920.

Nocentini, A., Menesini, E., \& Pluess, M. (2018, Nov). The Personality Trait of Environmental Sensitivity Predicts Children's Positive Response to School-Based Antibullying Intervention. Clinical Psychological Science, 6(6), 848-859. https://doi.org/10.1177/2167702618782194

Nolvi, S., Rasmussen, J. M., Graham, A. M., Gilmore, J. H., Styner, M., Fair, D. A., Entringer, S., Wadhwa, P. D., \& Buss, C. (2020, 2020/10/01/). Neonatal brain volume as a marker of differential susceptibility to parenting quality and its association with neurodevelopment across early childhood. Developmental Cognitive Neuroscience, 45, 100826. https://doi.org/https://doi.org/10.1016/j.den.2020.100826

Nylund, K. L., Asparouhov, T., \& Muthén, B. O. (2007). Deciding on the number of classes in latent class analysis and growth mixture modeling: A Monte Carlo simulation study. Structural Equation Modeling, 14(4), 535-569.

Obradovic, J., \& Boyce, W. T. (2009). Individual differences in behavioral, physiological, and genetic sensitivities to contexts: implications for development and adaptation. Developmental Neuroscience, 31(4), 300-308. 
Pluess, M. (2015). Individual Differences in Environmental Sensitivity. Child Development Perspectives, 9(3), 138-143. https://doi.org/10.1111/cdep.12120

Pluess, M., Assary, E., Lionetti, F., Lester, K. J., Krapohl, E., Aron, E. N., \& Aron, A. (2018, Jan). Environmental sensitivity in children: Development of the Highly Sensitive Child Scale and identification of sensitivity groups. Developmental Psychology, 54(1), 51-70. https://doi.org/10.1037/dev0000406

Pluess, M., \& Belsky, J. (2010). Children's Differential Susceptibility to Effects of Parenting. Family Science, 1(1), 14-25.

Pluess, M., \& Belsky, J. (2011, Feb). Prenatal programming of postnatal plasticity? Development and Psychopathology, 23(1), 29-38. https://doi.org/10.1017/S0954579410000623

Pluess, M., \& Belsky, J. (2013, Jul). Vantage sensitivity: Individual differences in response to positive experiences. Psychological Bulletin, 139(4), 901-916. https://doi.org/10.1037/a0030196

Pluess, M., Belsky, J., Way, B. M., \& Taylor, S. E. (2010, Aug 16). 5-HTTLPR moderates effects of current life events on neuroticism: differential susceptibility to 
environmental influences. Progress in Neuro-Psychopharmacology and Biological Psychiatry, 34(6), 1070-1074. https://doi.org/10.1016/j.pnpbp.2010.05.028

Pluess, M., \& Boniwell, I. (2015, 8//). Sensory-Processing Sensitivity predicts treatment response to a school-based depression prevention program: Evidence of Vantage Sensitivity. Personality and Individual Differences, 82(0), 40-45. https://doi.org/http://dx.doi.org/10.1016/j.paid.2015.03.011

Pluess, M., De Brito, S., Jones, A. P., McCrory, E., \& Viding, E. (in press). Individual Differences in Sensitivity to the Early Environment as a Function of Amygdala and Hippocampus Volumes: An Exploratory Analysis in 12-Year Old Boys. Development and Psychopathology.

Pluess, M., Rhoades, G. K., Keers, R., Knopp, K., Belsky, J., Markman, H. J., \& Stanley, S. M. (submitted). Genetic Sensitivity Predicts Long-Term Psychological Benefits of a Relationship Education Program for Married Couples.

Pornprasertmanit, S., Miller, P., Schoemann, A., \& Rosseel, Y. (2013). semTools: Useful tools for structural equation modeling. $R$ package version $0.4-14$.

Power, R. A., \& Pluess, M. (2015, 07/14/online). Heritability estimates of the Big Five personality traits based on common genetic variants [Original Article]. Transl Psychiatry, 5, e604. https://doi.org/10.1038/tp.2015.96 
Preacher, K. J., Rucker, D. D., MacCallum, R. C., \& Nicewander, W. A. (2005). Use of the extreme groups approach: a critical reexamination and new recommendations. Psychological Methods, 10(2), 178.

Rosseel, Y. (2012). An R Package for Structural Equation Modeling. Journal of Statistical Software, 48(2), 1-36. http://cran.r-project.org/web/packages/lavaan/lavaan.pdf

Sanchez-Roige, S., Gray, J. C., MacKillop, J., Chen, C. H., \& Palmer, A. A. (2018, Mar). The genetics of human personality. Genes, Brain and Behavior, 17(3), e12439. https://doi.org/10.1111/gbb.12439

Satorra, A. (2000). Scaled and adjusted restricted tests in multi-sample analysis of moment structures. In Innovations in multivariate statistical analysis (pp. 233-247). Springer.

Schermelleh-Engel, K., Moosbrugger, H., \& Müller, H. (2003). Evaluating the fit of structural equation models: Tests of significance and descriptive goodness-of-fit measures. Methods of Psychological Research Online, 8(2), 23-74.

Schriber, R. A., Anbari, Z., Robins, R. W., Conger, R. D., Hastings, P. D., \& Guyer, A. E. (2017). Hippocampal Volume as an Amplifier of the Effect of Social Context on Adolescent Depression. Clinical Psychological Science, 5(4), 632-649. https://doi.org/10.1177/2167702617699277 
Slagt, M., Dubas, J. S., Dekovic, M., \& van Aken, M. A. (2016, Oct). Differences in sensitivity to parenting depending on child temperament: A meta-analysis. Psychological Bulletin, 142(10), 1068-1110. https://doi.org/10.1037/bul0000061

Slagt, M., Dubas, J. S., van Aken, M. A. G., Ellis, B. J., \& Dekovic, M. (2018, Mar). Sensory Processing Sensitivity as a Marker of Differential Susceptibility to Parenting. Developmental Psychology, 54(3), 543-558. https://doi.org/10.1037/dev0000431

Smolewska, K. A., McCabe, S. B., \& Woody, E. Z. (2006, Apr). A psychometric evaluation of the Highly Sensitive Person Scale: The components of sensory-processing sensitivity and their relation to the BIS/BAS and "Big Five". Personality and Individual Differences, 40(6), 1269-1279. https://doi.org/10.1016/j.paid.2005.09.022

van IJzendoorn, M. H., Belsky, J., \& Bakermans-Kranenburg, M. J. (2012). Serotonin transporter genotype 5HTTLPR as a marker of differential susceptibility? A metaanalysis of child and adolescent gene-by-environment studies. Translational psychiatry, 2, e147. https://doi.org/10.1038/tp.2012.73

Wagenmakers, E.-J., \& Farrell, S. (2004). AIC model selection using Akaike weights. Psychonomic bulletin \& review, 11(1), 192-196. 
Whittle, S., Yap, M. B. H., Sheeber, L., Dudgeon, P., Yuecel, M., Pantelis, C., Simmons, J. G., \& Allen, N. B. (2011, Feb). Hippocampal volume and sensitivity to maternal aggressive behavior: A prospective study of adolescent depressive symptoms. Development and Psychopathology, 23(1), 115-129. https://doi.org/10.1017/s0954579410000684

Wolf, M., van Doorn, G. S., \& Weissing, F. J. (2008, Oct 14). Evolutionary emergence of responsive and unresponsive personalities. Proceedings of the National Academy of Sciences of the United States of America, 105(41), 15825-15830. https://doi.org/10.1073/pnas.0805473105

Yap, M. B., Whittle, S., Yucel, M., Sheeber, L., Pantelis, C., Simmons, J. G., \& Allen, N. B. (2008, Dec). Interaction of parenting experiences and brain structure in the prediction of depressive symptoms in adolescents. Archives of General Psychiatry, 65(12), 1377-1385. https://doi.org/10.1001/archpsyc.65.12.1377 


\section{Tables}

\section{Table 1}

Items included in the HSP-12 scale.

Item

Subscale

1. Do you seem to be aware of subtleties in your environment?

AES

2. Are you easily overwhelmed by things like bright lights, strong

LST

smells, coarse fabrics, or sirens close by?

3. Do you have a rich, complex inner life?

AES

4. Do you get rattled when you have a lot to do in a short amount of

EOE time?

5. Are you deeply moved by the arts or music?

6. Are you annoyed when people try to get you to do too many

EOE things at once?

7. Do you make a point to avoid violent movies and TV shows?

8. Do you find it unpleasant to have a lot going on at once?

EOE

9. Do changes in your life shake you up?

EOE

10. Do you notice and enjoy delicate or fine scents, tastes, sounds,

AES works of art?

11. Are you bothered by intense stimuli, like loud noises or chaotic scenes?

12. When you must compete or be observed while performing a task,

EOE do you become so nervous or shaky that you do much worse than you would otherwise?

Note. EOE = Ease of Excitation; AES = Aesthetic Sensitivity; LST = Low Sensory Threshold. 
Table 2

Bivariate associations between HSP-12 total and subscales with Big Five personality dimensions and facets (Study 1, N = 722).

\begin{tabular}{|c|c|c|c|c|}
\hline & HSP-12 & 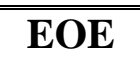 & AES & LST \\
\hline Neuroticism & $.51^{* * *}$ & $.68^{* * *}$ & -.04 & $.37^{* *}$ \\
\hline Anxiety & $.52^{* *}$ & $.64^{* * *}$ & .00 & $.39^{* *}$ \\
\hline Anger & $.24^{* *}$ & $.37^{* *}$ & $-.09^{*}$ & $.16^{* *}$ \\
\hline Depression & $.37^{* *}$ & $.50^{* *}$ & -.02 & $.25^{* *}$ \\
\hline Self-consciousness & $.39^{* *}$ & $.53^{* *}$ & -.07 & $.30^{* *}$ \\
\hline Immoderation & .06 & $.09^{*}$ & .02 & -.01 \\
\hline Vulnerability & $.55^{* *}$ & $.68^{* *}$ & -.00 & $.42^{* *}$ \\
\hline Extraversion & $-.30^{* *}$ & $-.47^{* *}$ & $.14^{* *}$ & $-.25^{* *}$ \\
\hline Friendliness & $-.36^{* *}$ & $-.48^{* *}$ & $.07^{*}$ & $-.30^{* *}$ \\
\hline Gregariousness & $-.33^{* *}$ & $-.40^{* *}$ & .04 & $-.30^{* *}$ \\
\hline Assertiveness & $-.18^{* *}$ & $-.32^{* *}$ & $.13^{* *}$ & $-.15^{* *}$ \\
\hline Activity level & -.07 & $-.17^{* *}$ & .08 & -.00 \\
\hline Excitement seeking & $-.12^{* *}$ & $-.21^{* *}$ & $.14^{* *}$ & $-.15^{* *}$ \\
\hline Cheerfulness & $-.19^{* *}$ & $-.36^{* *}$ & $.16^{* *}$ & $-.13^{* *}$ \\
\hline Openness & $.25^{* *}$ & -.06 & $.63^{* *}$ & $.07^{*}$ \\
\hline Imagination & $.33^{* *}$ & $.16^{* *}$ & $.40^{* *}$ & $.19^{* *}$ \\
\hline Artistic interests & $.30^{* *}$ & -.02 & $.64^{* *}$ & $.12^{* *}$ \\
\hline Emotionality & $.31^{* *}$ & .17 & $.34^{* *}$ & $.19^{* *}$ \\
\hline Adventurousness & $-.23^{* *}$ & $-.43^{* *}$ & $.22^{* *}$ & $-.20^{* *}$ \\
\hline Intellect & .04 & -.15 & $.41^{* *}$ & -.08 \\
\hline Liberalism & $.10^{*}$ & .03 & $.19^{*}$ & .01 \\
\hline
\end{tabular}




\begin{tabular}{lcccc}
\hline \hline Agreeableness & .02 & -.03 & $.11^{*}$ & -.00 \\
Trust & $-.19^{* *}$ & $-.23^{* *}$ & -.02 & $-.14^{* *}$ \\
Morality & -.01 & -.06 & .08 & -.04 \\
Altruism & $.12^{*}$ & .01 & $.23^{* *}$ & .05 \\
Cooperation & .01 & -.05 & .06 & .04 \\
Modesty & .06 & $.17^{* *}$ & $-.12^{*}$ & .05 \\
Sympathy & $.15^{* *}$ & .04 & $.27^{* *}$ & .04 \\
Conscientiousness & $-.15^{* *}$ & $-.27^{* *}$ & $.08^{*}$ & $-.08^{*}$ \\
Self-efficacy & $-.20^{* *}$ & $-.34^{* *}$ & $.14^{* *}$ & $-.16^{* *}$ \\
Orderliness & $-.11^{*}$ & -.14 & -.03 & -.04 \\
Dutifulness & -.02 & -.06 & .01 & -.01 \\
Achievement striving & -.06 & $-.20^{* *}$ & $.16^{* *}$ & -.04 \\
Self - discipline & $-.20^{* *}$ & $-.32^{* *}$ & .05 & -.10 \\
Cautiousness & -.03 & -.07 & .03 & -.01 \\
\hline \hline
\end{tabular}

Note. EOE = Ease of Excitation; AES = Aesthetic Sensitivity; LST = Low Sensitivity Threshold; Associations that were tested for divergent validity are marked bold (i.e., all correlation with $r=.50$ or higher); ${ }^{*} p<.05 ;{ }^{* *} p<.01$ 


\section{Table 3}

Identification of Sensitivity Groups with Latent Class Analysis (Study 2, N = 952).

\begin{tabular}{|c|c|c|c|c|c|}
\hline Classes & AIC & $\overline{\mathrm{BIC}}$ & BIC adj. & LMR-A $(p)$ & Entropy \\
\hline One & 43248.237 & 43364.843 & 43288.620 & - & - \\
\hline Two & 41472.101 & 41651.868 & 41534.357 & $1782.149(p<.001)$ & .83 \\
\hline Three & 41048.975 & 41291.903 & 41133.105 & $444.145(p=. \underline{016})$ & .77 \\
\hline \multirow[t]{2}{*}{ Four } & 40794.159 & 41100.249 & 40900.163 & $277.701 \quad(p=$ & .78 \\
\hline & & & & $.243 e t)$ & \\
\hline Five & 40629.780 & 40999.031 & 40757.658 & $188.268(p=.107)$ & .77 \\
\hline Six & 40475.976 & $40908 . \underline{388}$ & 40625.728 & $177.809(p=. \underline{182})$ & .80 \\
\hline
\end{tabular}

Note. The solution with the overall best fit with is marked bold. 


\section{Table 4}

Bivariate correlations between all variables (Study 3, $N=110$ ).

$\begin{array}{llllllll}1 & 2 & 3 & 4 & 5 & 6 & 7 & 8\end{array}$

1. Gender (1= male, $2=$ female $)$

2. Age

$.03-$

3. HSP-12

$.34^{* *}-.02$

4. Wellbeing Baseline

$\begin{array}{lll}-.16 & -.08 & -.08\end{array}$

5. Wellbeing FU1

$\begin{array}{llll}.17 & -.06 & .06 & .40^{* *}\end{array}$

6. Wellbeing FU2

$\begin{array}{llllll}-.20 & .09 & \mathbf{- . 3 7 ^ { * * }} & .55^{* *} & \mathbf{. 8 2} & \text { ** }\end{array}$

7. Wellbeing FU3

$\begin{array}{llllll}-.11 & -.03 & -.18 & .52^{* *} & .60^{* *} & .77^{* *}\end{array}$

8. Wellbeing FU4

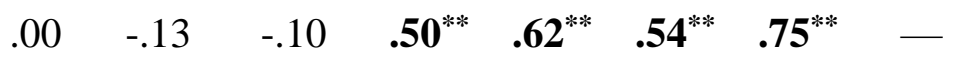

9. Wellbeing FU5

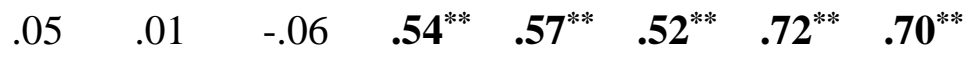

Note. Statistically significant correlations are marked bold; ${ }^{*} p<.05 ;{ }^{* *} p<.01$. 


\section{Table 5}

Bivariate correlations between all variables (Study $4, N=80)$.

$\begin{array}{lllll}1 & 2 & 3 & 4 & 5\end{array}$

1. Gender $(0=$ male, $1=$ female $)$

2. Age

.00

3. HSP-12

$.14 \quad .18$

4. Positive Mood before video-clip $\quad .01$

$.02-.17$

5. Positive Mood after video-clip

$.27^{*}$

.03

.08

$.71^{* *}$

Note. Statistically significant correlations are marked bold; ${ }^{*} p<.05 ;{ }^{* *} p<.01$. 
Table 6

Comparison of mixed-effect regression models predicting pre-post change in positive mood (Study 4).

\begin{tabular}{lcc}
\hline \hline Models & Chisq $(\boldsymbol{p})$ & AIC weights \\
\hline Model 1: Gender & - & .00 \\
Model 2: Gender, Time & $91.71(p<.01)$ & .04 \\
Model 3: Gender, Time, HSP-12 & $.55(p=.46)$ & .02 \\
Model 4: Gender, Time, Time x HSP-12 & $9.68(p<.01)$ & .94 \\
\hline \hline
\end{tabular}




\section{Figure Legends}

\section{Figure 1}

Graphic illustration of the bifactor structure consisting of one general sensitivity factor (HSP) on which all items load as well as three individual factors with five items loading onto Ease of Excitation (EOE), four items loading onto Aesthetic Sensitivity (AES), and three items loading onto Low Sensory Threshold (LST).

\section{Figure 2}

Distribution of the three sensitivity groups that were identified through Latent Class Analysis and intersection points between the detected groups with 3.87 between low and medium sensitivity and 4.63 between medium and high sensitivity groups.

\section{Figure 3}

Change in self-reported well-being of teacher trainees across six time points, from baseline to follow-up 5, separately for participants scoring low (i.e., bottom 30\%) versus high (i.e., top $30 \%$ ) in sensitivity measured with the HSP-12. The lines represent estimated values for low and high sensitivity groups and shaded areas represent confidence interval.

\section{Figure 4}

Change in self-reported happiness of study participants from before to after watching a video of uplifting nature, separately for participants scoring low (i.e., bottom 30\%) versus high (i.e., top 30\%) in sensitivity measured with the HSP-12. The lines represent estimated values for the low and high sensitivity groups and shaded areas represent standard errors. 
Figure 1

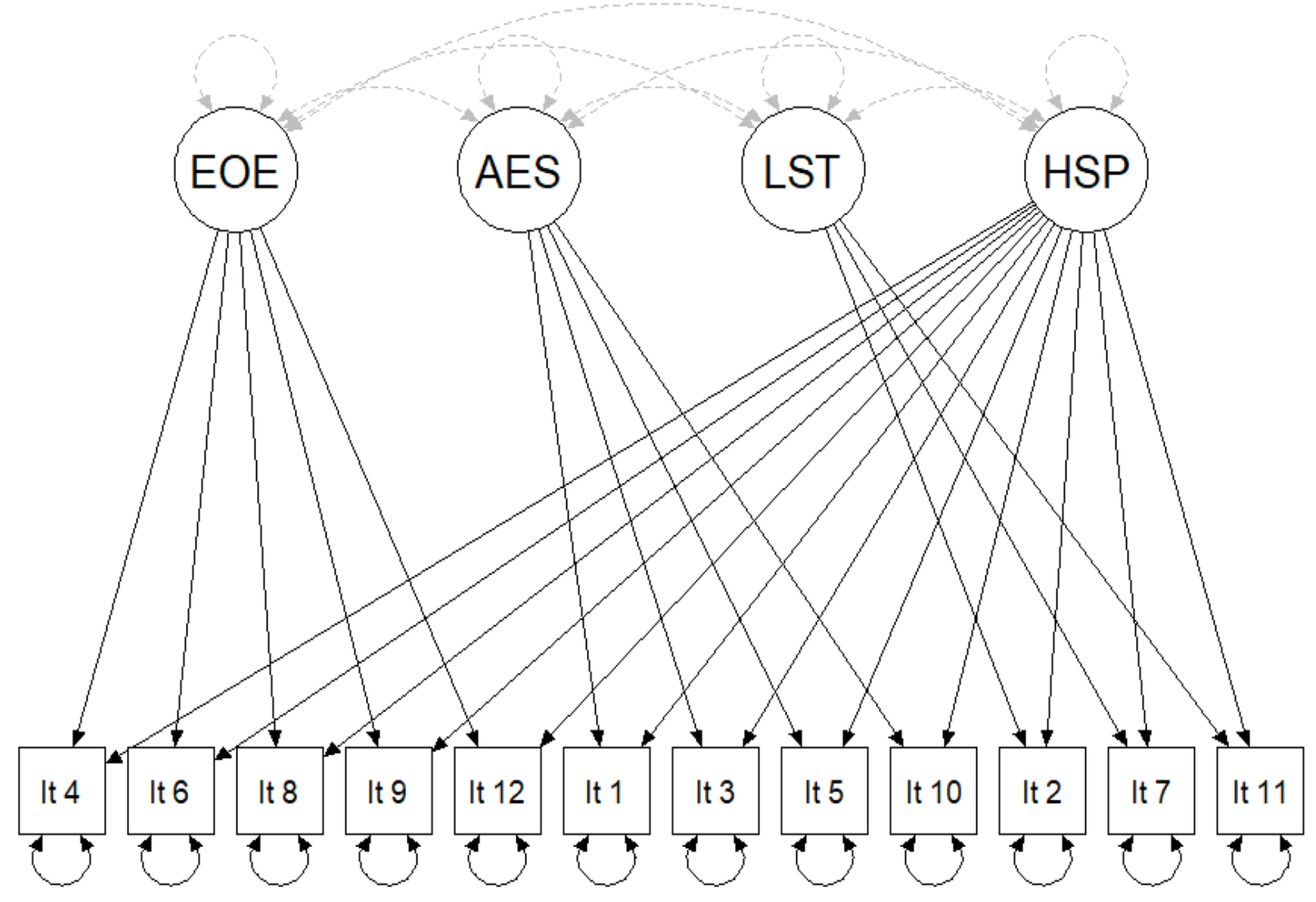


Figure 2

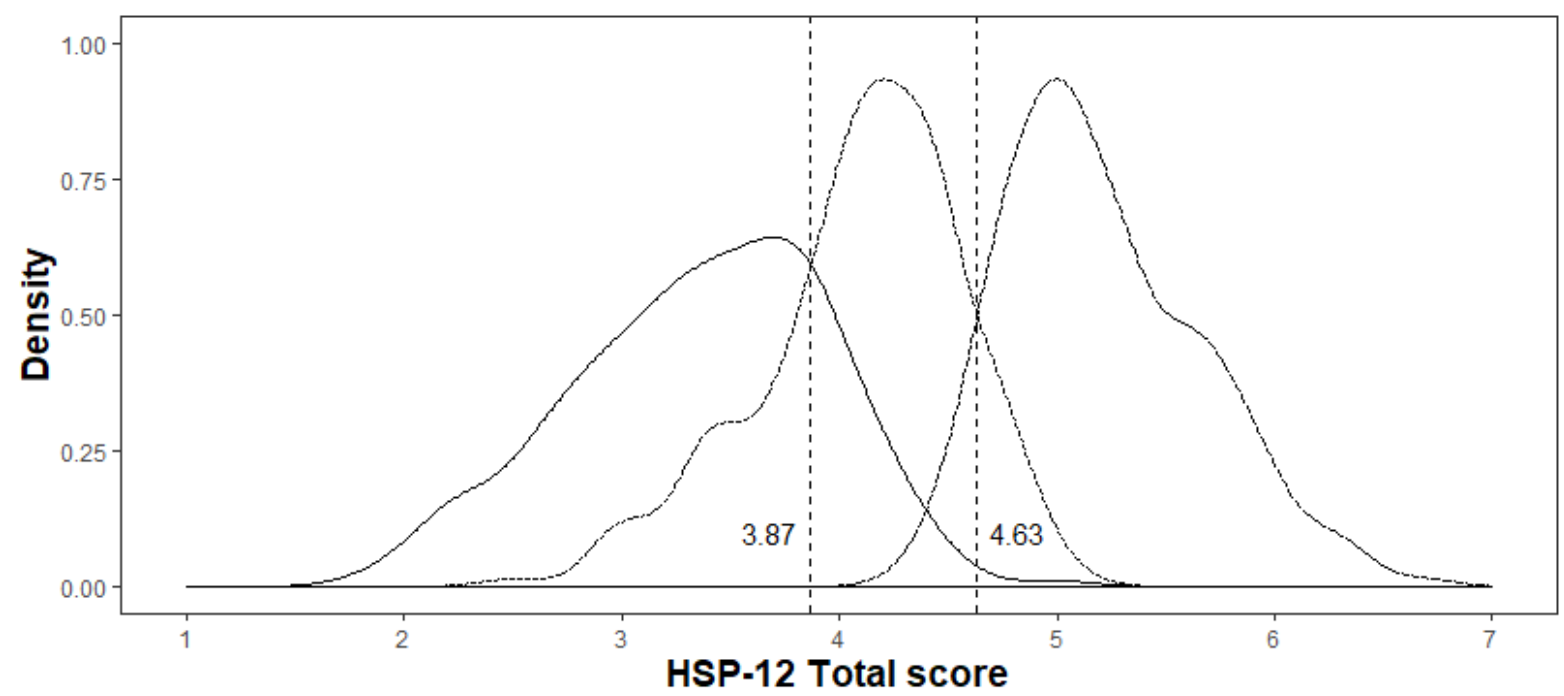


Figure 3

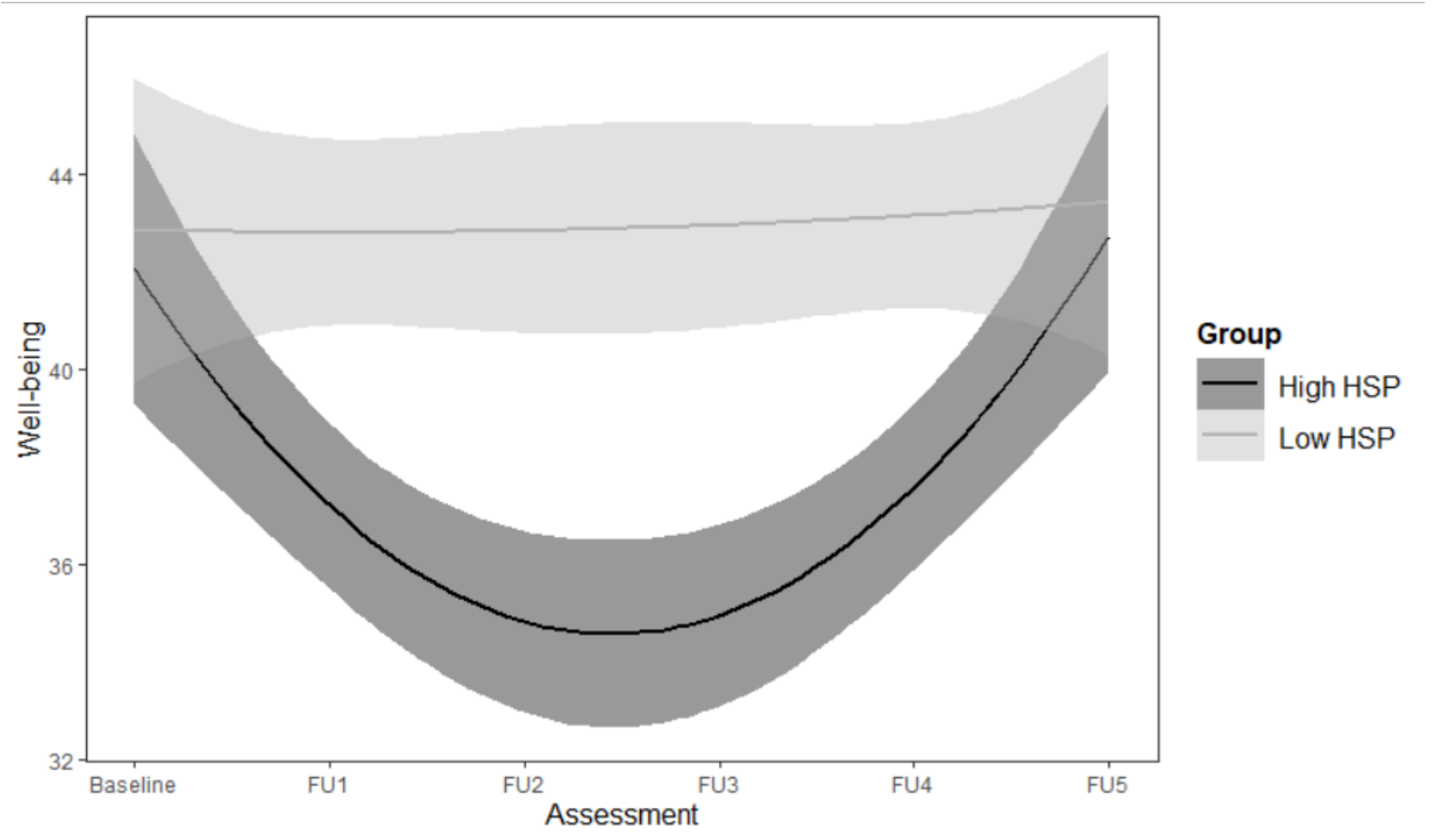


Figure 4

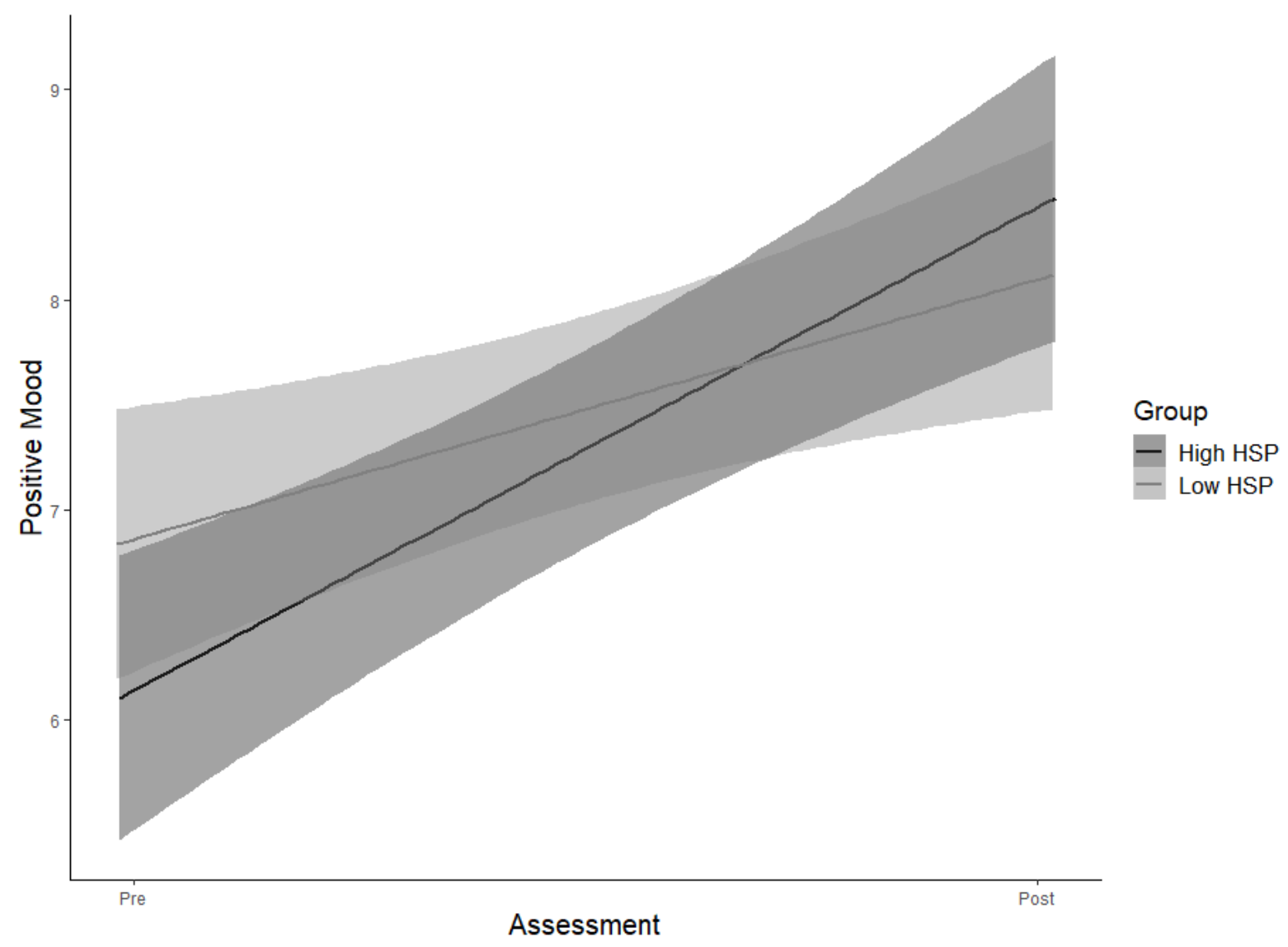

